\title{
Arrhythmogenic ion-channel remodeling in heart failure
}

\author{
Norbert Jost ${ }^{1 *}$, Danina Muntean ${ }^{2}$ \\ ${ }^{1}$ Hungarian Academy of Sciences affiliated to Department of Pharmacology \& Pharmacotherapy, Faculty \\ of Medicine, University of Szeged, Szeged, Hungary \\ ${ }^{2}$ University of Medicine and Pharmacy, Timisoara, Romania
}

Heart failure carries a poor prognosis, namely, about a half of the affected patients die within five years after the initial diagnosis, and a half of these deaths are due to severe arrhythmia. The underlying proarrhythmic mechanisms in the heart failure consist of abnormal excitability and impulse conduction resulting in a triggered activity, reentrant wave fronts and/or rotors, that are related to aberrant intracellular $\mathrm{Ca}_{2}+$-cycling. Cardiac disease modifies the operation of ion channels and transporters in a way that promotes the occurrence of cardiac rhythm disturbances, the process called "arrhythmogenic remodeling". Arrhythmogenic remodeling involves alterations in ion channel and transporter expression, regulation and association with important protein part-

Received: $27^{\text {th }}$ Apr 2014

*Address for correspondence: Division of Cardiovascular Pharmacology, Hungarian Academy of Sciences affiliated to Department of Pharmacology \& Pharmacotherapy, Faculty of Medicine, University of Szeged, Dom ter 12, H-6720 Szeged, Hungary.

Phone: +36-62-546-885

E-mail: jost.norbert@gmail.com ners and has important pathophysiological implications that in major ways contribute to cardiac morbidity and mortality. We review the changes in ion channel and transporter properties associated with the congestive heart failure. We pay a particular attention to $\mathrm{K}, \mathrm{Na}$ and $\mathrm{Ca}$ channels; $\mathrm{Ca}$ transporters; connexins; and hyperpolarization-activated nonselective cation channels and discuss the mechanisms through which the changes in ion handling processes lead to cardiac arrhythmias. We highlight the areas of future investigation, important opportunities for improved therapeutic approaches that are being opened by an improved understanding of the mechanisms of arrhythmogenic remodeling.

This paper was supported by HU-RO Cross-Border Cooperation Programme (HURO/1101/086/2.2.1_HURO-TWIN), and by the National Research Council Project IDEAS - Programme "Exploratory Research Projects" PN-II-ID-PCE-2012-4-0512.

KEYWORDS: arrythmogenic, ion-channel, remodelling, heart, failure.

CITATION: Cardiol Croat. 2014;9(5-6):213. 